\title{
Simultaneous Realization of Wavelength Conversion, 2R Regeneration, and All-Optical Multiple Logic Gates with OR, NOR, XOR, and XNOR Functions Based on Self-Polarization Rotation in a Single SOA: An Experimental Approach
}

\author{
Youssef Said and Houria Rezig \\ Sys'com Lab, National Engineering School of Tunis (ENIT), B.P. 37, Le Belvedere, 1002 Tunis, Tunisia \\ Correspondence should be addressed to Youssef Said, youssef.said@tunisietelecom.tn
}

Received 31 October 2011; Revised 29 January 2012; Accepted 30 January 2012

Academic Editor: Yu S. Kivshar

Copyright ( 2012 Y. Said and H. Rezig. This is an open access article distributed under the Creative Commons Attribution License, which permits unrestricted use, distribution, and reproduction in any medium, provided the original work is properly cited.

\begin{abstract}
We highlight the feasibility of experimental implementation of both inverted and noninverted wavelength conversion, $2 \mathrm{R}$ regeneration, and all-optical logic functions, such as OR, NOR, XOR, and XNOR optical gates by exploiting the self-polarization rotation in a semiconductor optical amplifier (SOA) device without changing the setup configuration. Switching between each optical function is done by only adjusting the input optical power level. In order to allow optimum control and preserve the polarization state of the injected and collected signals, the polarimetric measures have been carried out in free space.
\end{abstract}

\section{Introduction and State of the Art}

Semiconductor optical amplifier (SOA) is a promising and fundamental component in today's photonic networks and next-generation optical networks. It is characterized by high nonlinearities, compactness, multifunctionality, and high ability of integration. It has proven to be a versatile and multifunctional device to be used to achieve different functions in access, core, and metropolitan networks. Particularly, it has been envisioned for all-optical signal processing tasks at very high bit rates, that cannot be handled by electronics, such as wavelength conversion [1-4], signal regeneration $[5,6]$, optical switching [7] and, optical logic operations [8$10]$.

All-optical wavelength converters and optical regenerators can be achieved by exploiting SOA nonlinearities such as cross-gain modulation (XGM) [11], cross-phase modulation (XPM) $[3,12,13]$, four-wave mixing (FWM) $[14,15]$, and cross-polarization modulation (XPolM) $[6,16,17]$. They have attracted a lot of interest thanks to their attractive features, such as small size, fast carrier dynamics, multifunctional aspect, power consumption, optical power efficiency, and high potential of integration. The main features of wavelength converters include their transparency to bit rate and signal format, operation at moderate optical power levels, low electrical power consumption, small frequency chirp, cascadability of multiple stages of converters, and signal reshaping.

All-optical wavelength converters at bit rates from 10 up to $100 \mathrm{Gbit} / \mathrm{s}$ were experimentally and theoretically investigated, by Leuthold et al., by using a fully integrated. SOA-delayed interference configuration [1]. Furthermore, Randhawa et al. [3] have simulated wavelength converter for future broadcast networks at $40 \mathrm{Gbit} / \mathrm{s}$ using low-cost SOAs. Their performance analysis is carried out for an all-optical frequency converter based on XPM in two SOAs arranged in a Mach-Zehnder interferometer (MZI) configuration to evaluate the efficiency of conversion. Their results show that conversion is possible over a wavelength separation of $1 \mathrm{~nm}$ between the pump and the input wavelength. They have demonstrated that increasing the driving current can decrease the XPM effect and the XGM scheme shows extinction ratio degradation for conversion to longer wavelengths [3]. 
In addition, Spyropoulou et al. [4] have presented theoretical and experimental performance analysis of $40 \mathrm{Gbit} / \mathrm{s}$ non-return-to-zero (NRZ) all-optical wavelength conversion using a differentially biased SOA-MZI. Their theoretically obtained results are confirmed through experiments that demonstrate successful $40 \mathrm{Gbit} / \mathrm{s}$ wavelength conversion functionality for NRZ data signals only when a differentially biased SOA-MZI configuration is employed, whereas an error floor is obtained when $40 \mathrm{Gbit} / \mathrm{s}$ NRZ all-optical wavelength conversion with the standard single-control SOAMZI scheme is attempted [4]. Moreover, Turkiewiez et al. [16] have reported all optical 1310 to $1550 \mathrm{~nm}$ wavelength conversion based on nonlinear polarization rotation in an $\mathrm{SOA}$, at bit rate $10 \mathrm{Gbit} / \mathrm{s}$, in between two transmission links by using two standard single-mode fiber-based spans.

Wavelength conversion based on FWM process in SOAs is an attractive technique, compared to XGM and XPM, since it is independent of modulation format, ultrafast, and capable of dispersion compensation. It offers strict transparency, including modulation-format and bit-rate transparency and is capable of multiwavelength conversions. However, it has low conversion efficiency and needs careful control of the input signal polarization. The main drawbacks of wavelength conversion based on FWM are polarization sensitivity and the frequency-shift dependent conversion efficiency. However, wavelength conversion based on XPolM is another promising approach. It uses the optically induced birefringence and dichroism in an SOA and has great potential to offer wavelength conversion with high extinction ratio.

Optical logic gates can be realized either by exploiting SOA nonlinearities, such as XGM [18-20], FWM [20, 21], and XPolM [10, 22, 23]. Berrettini et al. [20] have demonstrated an integrable scheme of reconfigurable and ultrafast photonic logic gate, based on a single SOA and able to process ultrafast signals. They have implemented XNOR function exploiting XGM and FWM in an SOA. They have showed that the same scheme can be easily reconfigured to obtain AND, NOR, and NOT logic gates [20].

Although the principle of all-optical gates, wavelength conversion, and $2 \mathrm{R}$ optical regeneration, which are based on nonlinear polarization rotation, has already been demonstrated by others authors [6, 10, 22-25], we propose and argue, in the next sections of this paper, a promising approach, which has not been reported yet according to our knowledge, of the implementation method of optical OR, NOR, XOR, and XNOR gates, wavelength converter, and $2 \mathrm{R}$ optical regenerator by exploiting the self-polarization rotation (SPR) in a SOA structure. The implementation of those functions was made by referring to the same setup configuration in free space that can allow optimum control and preservation of the polarization state of the injected and collected signals. Switching between each optical function is done by only adjusting the input optical power level.

\section{Presentation of the Experimental Setup}

For allowing optimum control and preservation of the polarization state of the injected and collected signals, the experimentation has been carried out in free space in the research laboratory in electronics, signal, optoelectronics and telecommunications (RESO), Brest National Engineering school (ENIB), France. We used a commercial, a bulk, and a tensile-strained SOA structure, having the reference: $1550 \mathrm{CRI} / \mathrm{P}-\mathrm{SN}$ 2106, which is manufactured by OptoSpeed. It is based on InP/GaInAsP, having an active layer length $L=500 \mu \mathrm{m}$, active zone width $W=2,5 \mu \mathrm{m}$, and an active layer height $d=0,2 \mu \mathrm{m}$. The experimental setup is shown in Figure 1. The SOA is placed in such a way that their TE and TM axes correspond, respectively, to the horizontal and vertical axes of the lab referential.

As the experiment was done in free space, the risk of errors is high. In order to reduce it, we adopted three calibration steps, which are

(i) the optical beams alignment,

(ii) the optical elements alignment,

(iii) the calibration of the bench polarimeter at light running.

Light emitted from the SOA was collected and collimated with a microscope objective, then passed through the equivalent of a polarization controller, which is formed with a quarter-wave plate (QWP) and a half-wave plate (HWP). Subsequently, it was passed through a linear polarizer (LP) acting as an analyzer. Then, it was recollected with a fibred collimator (FC) that is connected to an optical spectrum analyzer (OSA), having a resolution of $0.07 \mathrm{~nm}$ in order to reject the amplified spontaneous emission (ASE) of the SOA. The passing axis of the linear polarizer, when set vertically, coincided with the TM axis in the sample and defined a reference direction from which the orientation $\theta$ of the fast axis of the quarter-wave plate was estimated. This orientation could be modified, as the quarter-wave plate was mounted on a rotation stage whose movements were accurately determined by a computer-controlled step motor.

In order to inject a linear polarization while assuring an equality of both TE and TM powers, the linear input polarizer was fixed to an angle $\theta=135^{\circ}$ with regard to the horizontal axis. The linearization was made with the output polarization controller, which consists of the QWP and the HWP, whereas the signal blocking was made with the output polarizer (LP) around a power, known as the blocking power. We have varied both QWP and HWP in order to obtain the lowest possible output power.

After several tests, we have chosen a SOA blocking power having a value equal to $-2 \mathrm{dBm}$, which is situated in the saturation regime interval of the device, because it allows to obtain a strong variation of the output power for a slight variation of the input power. This value seems to be the best compromise to optimize the static performances of the optical signal processing functions. Indeed, it allows a very good improvement of the extinction rate of the injected signal. The evolution of the transfer function of the SOA after blocking the output signal at an input power equal to $P_{\text {in }}=-2 \mathrm{dBm}$ for a bias current equal to $150 \mathrm{~mA}$ and $200 \mathrm{~mA}$ is illustrated in Figure 2. 


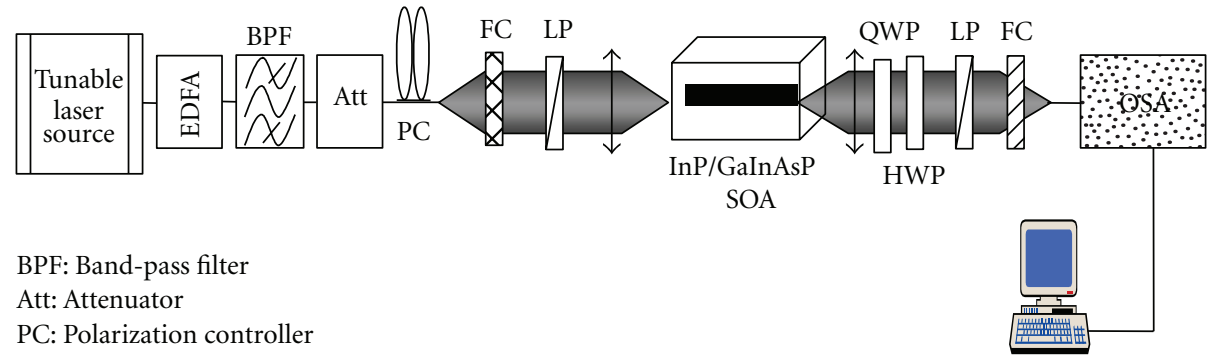

FIGURE 1: Experimental setup.

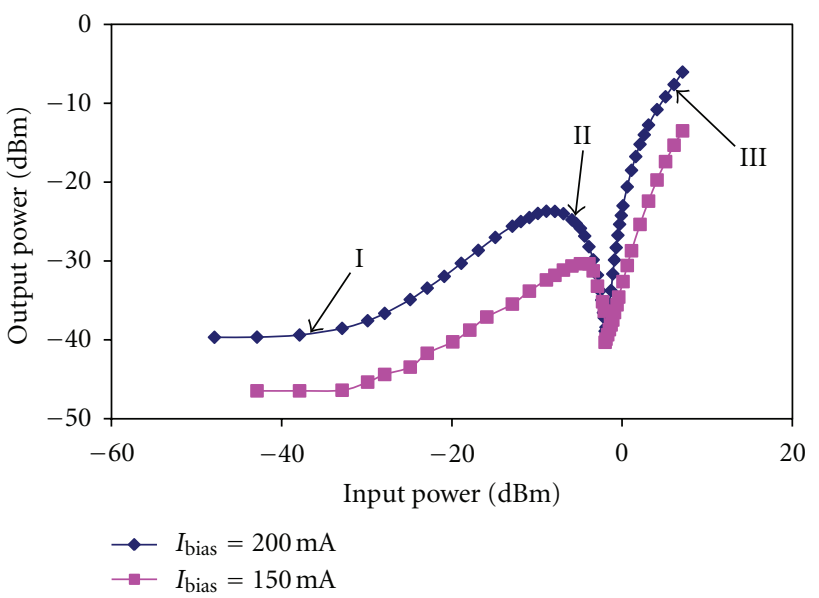

FIGURE 2: Measured static transfer function of the SOA at a blocking power equal to $-2 \mathrm{dBm}$ and wavelength equal to $1550 \mathrm{~nm}$ for a bias current $I_{\text {bias }}$ equal to $150 \mathrm{~mA}$ and $200 \mathrm{~mA}$.

We notice that the SOA output power takes much more significant values when the injected current increases which corresponds to a low contribution of the ASE. We can also note that the curve of the measured static transfer function of the SOA can show three various regimes according to the injected optical power. The first regime corresponds to a "slow" increase of the output power by increasing the input power. The second regime makes reference to a fast diminution of the output power further to the blocking. In the third regime, the SOA output power becomes more and more important with the increase of the injected optical power.

\section{Experimental Implementation of both Inverted and Noninverted Wavelength Conversion and 2R Optical Regeneration}

All-optical wavelength conversion refers to the operation that consists of the transfer of the information carried in one wavelength channel to another wavelength channel in optical domain. It is a key requirement for optical networks, since it has basically to be used to extend the degree of freedom to the wavelength domain. Moreover, All-optical wavelength conversion is also indispensable in future optical packet

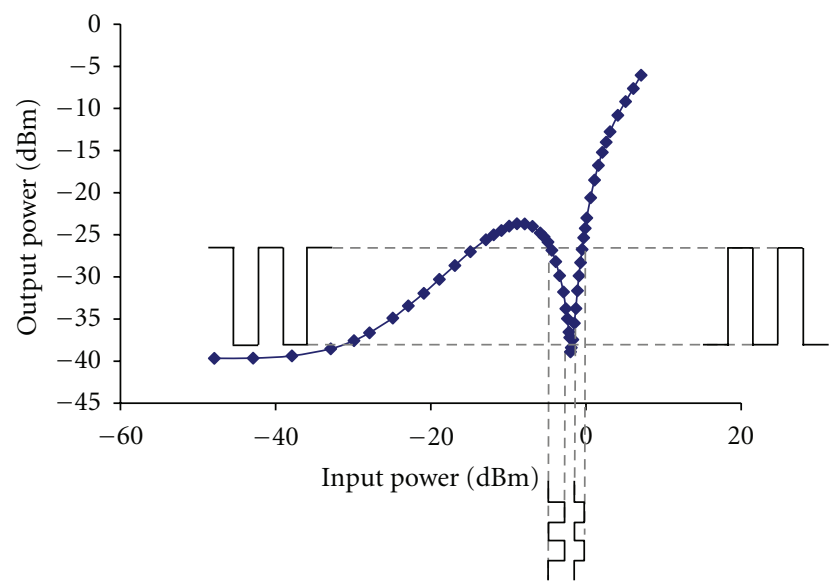

FIGURE 3: Measured static transfer function and principle of operation for both inverted and noninverted wavelength conversion at a blocking power equal to $-2 \mathrm{dBm}$ and wavelength equal to $1550 \mathrm{~nm}$.

switching networks to optimize the network performance metrics. It is very useful in the implementation of switches in WDM networks. In addition, it is crucial to lower the access blocking probability and therefore increase the utilization efficiency of the network resources in wavelength routed optical networks.

Referring to Figure 3, we can underline that by exploiting the nonlinear rotation of polarization in a single SOA, we can realize both inverted and noninverted wavelength conversion according to the choice of the average power value of the signal to be injected (pump). Indeed, if the value of this last one is lower than the blocking power, an inverted conversion wavelength is achieved. For the opposite case, a noninverted conversion is accomplished.

According to Figure 4, we can notice that the output extinction ratio is higher than the input extinction ratio $\left(E R_{\text {in }}<E R_{\text {out }}\right)$. This result allows us to note that by exploiting the self-polarization rotation, it is possible to accomplish $2 \mathrm{R}$ optical regeneration of a signal. The improvement of the extinction ratio is about $11 \mathrm{~dB}$ if the input power is fixed to $0 \mathrm{dBm}$. To benefit from the extinction ratio improvement, the power corresponding to the low level of the signal to be regenerated must be slightly superior to the blocking power and the power referring to its high level must not be very high, in order to limit the SOA saturation phenomenon. 


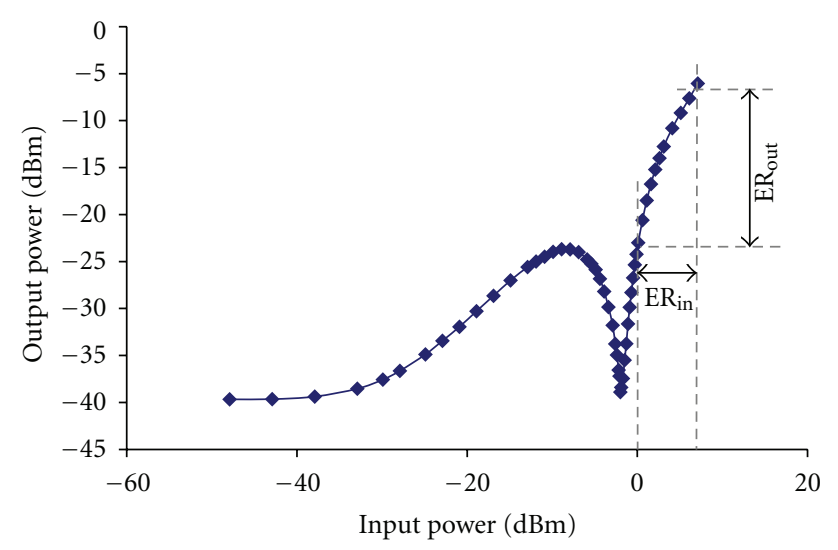

Figure 4: Measured static transfer function and principle of operation for $2 \mathrm{R}$ optical regeneration at a blocking power equal to $-2 \mathrm{dBm}$ and wavelength equal to $1550 \mathrm{~nm}$.

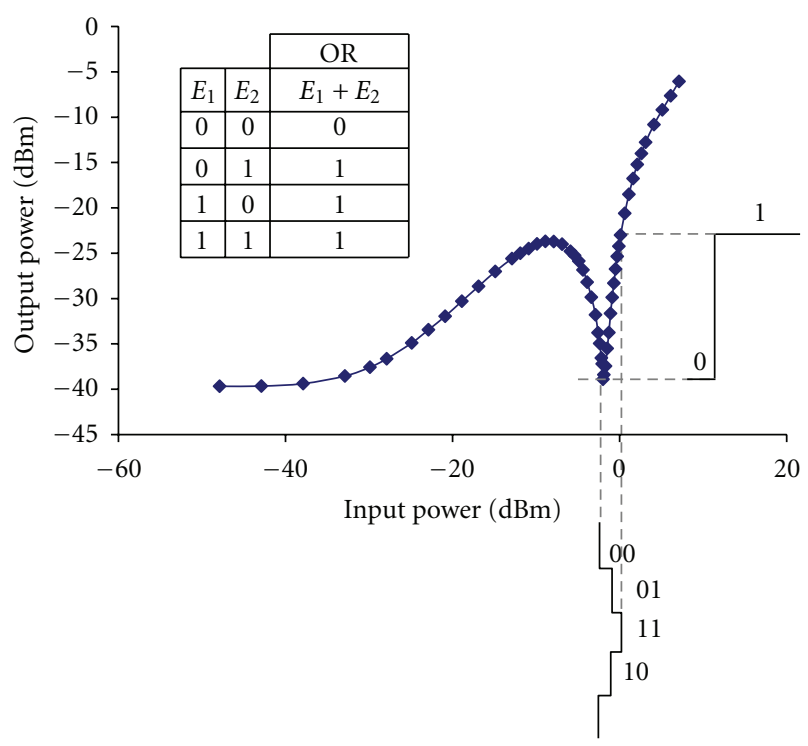

FIGURE 5: Measured static transfer function and principle of operation for OR function at a blocking power equal to $-2 \mathrm{dBm}$ and wavelength equal to $1550 \mathrm{~nm}$.

\section{Experimental Implementation of All-Optical Multiple Logic Gates}

4.1. Concept of All-Optical OR Logic Gate Implementation. Figure 5 exhibits the measured static transfer function of the OR gate that can be achieved and implemented using the same experimentation based on SPR.

The principle of operation for OR function is as follows: we consider that the pump signal is composed of signals: $E_{1}$ and $E_{2}$, which are simulated as logical entries for the logical gate. The output probe signal $\left(E_{s}=E_{1}+E_{2}\right)$ of the device serves as logical output. The three signals: $E_{1}, E_{2}$, and $E_{s}$ are simultaneously injected in the SOA. Then, the output stage, in the setup experimentation, is adjusted to block the signal when both pump signals are in their minimum power level, which corresponds to the low logic level (00). Consequently,

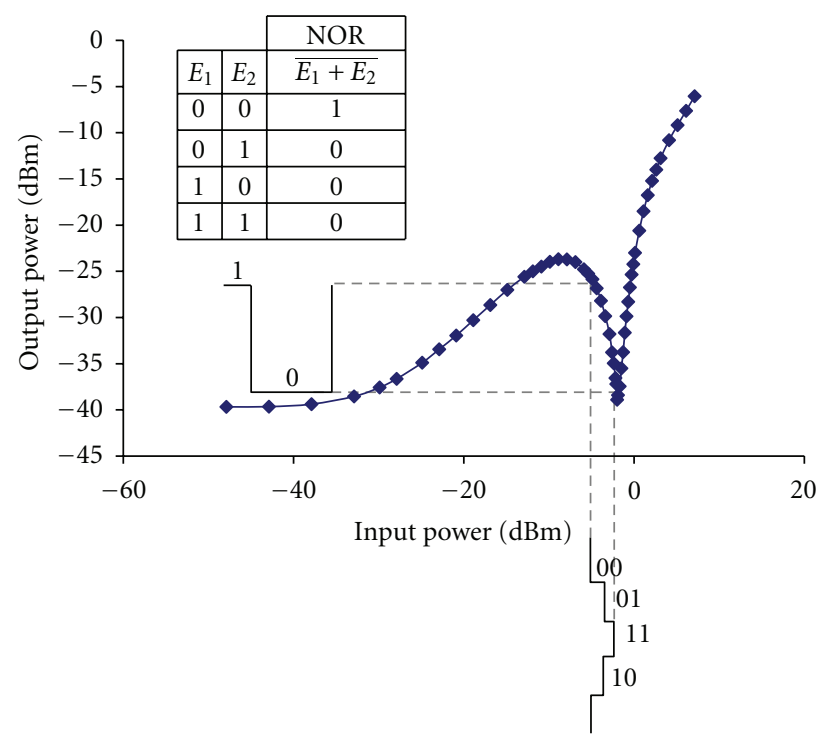

FIGURE 6: Measured static transfer function and principle of operation for NOR function at a blocking power equal to $-2 \mathrm{dBm}$ and wavelength equal to $1550 \mathrm{~nm}$.

the output logic level is low (0). Other cases correspond to the high logic level (1). So, the achievement of the optical OR logic gate is completed.

4.2. Concept of All-Optical NOR Gate Implementation. Referring to Figure 6, we can note that the optical NOR function can be achieved and implemented. Its functioning principle is the following: we assume that the pump signal is composed of signals: $E_{1}$ and $E_{2}$, which are considered as logical entries for the logical gate. The output probe signal $\left(E_{s}=\overline{E_{1}+E_{2}}\right)$ of the device serves as logical output. The three signals: $E_{1}$, $E_{2}$, and $E_{s}$ are simultaneously injected in the SOA. Then, the output stage, in the setup experimentation, is adjusted to block the signal when one among both pump signals is in its maximum power level, which corresponds to the high logic level $(01,10,11)$. As a result, the output logic level is low (0). The other case corresponds to the high logic level (1). Consequently, the same experimentation serves to accomplish the optical NOR logic gate implementation.

4.3. Concept of All-Optical XOR Gate Implementation. Figure 7 displays the measured static transfer function of the optical XOR gate that can be achieved and implemented using SPR. Its functioning principle is the following: the pump signal is assumed to be composed of signals: $E_{1}$ and $E_{2}$, which are considered as logical entries for the logical gate. The output probe signal $\left(E_{S}=E_{1} \cdot \overline{E_{2}}+\overline{E_{1}} \cdot E_{2}\right)$ of the device serves as logical output. The three signals: $E_{1}$, $E_{2}$, and $E_{s}$ are simultaneously injected in the SOA. Then, the output stage, in the setup experimentation, is adjusted to block the signal when both pump signals are in their maximum power level or when they are in their minimum power level, which corresponds, respectively, to the high logic levels (11) and (00). As a result, the output logic level is 




FIGURE 7: Measured static transfer function and principle of operation for XOR function at a blocking power equal to $-2 \mathrm{dBm}$ and wavelength equal to $1550 \mathrm{~nm}$.

low (0). The other case corresponds to the high logic level (1). As a result, the achievement of the optical XOR logic function implementation is obtained by adopting the same experimental setup.

4.4. Concept of All-Optical XNOR Gate Implementation. Referring to the measured static transfer function, which is presented in Figure 8, we can notice that XNOR gate can be achieved and implemented. Its principle of operation is the following: we consider that the pump signal is composed of signals: $E_{1}$ and $E_{2}$, which are playing the role of logical entries for the logical gate. The output probe signal $\left(E_{S}=\right.$ $\overline{E_{1} \cdot \overline{E_{2}}+\overline{E_{1}} \cdot E_{2}}$ ) of the device serves as logical output. The three signals: $E_{1}, E_{2}$, and $E_{s}$ are simultaneously injected in the SOA. Then, the output stage, in the setup experimentation, is adjusted to block the signal when only one pump signal is in its maximal power, which corresponds to the high logic levels (01 and 10). As a result, the output logic level is low (0). Other cases correspond to the high logic level (1). This case corresponds to the fulfillment of the optical XNOR logic gate.

\section{Conclusion}

We have proposed and experimentally demonstrated the achievement of wavelength converter, $2 \mathrm{R}$ optical regenerator and optical OR, NOR, XOR, and XNOR logic gates by exploiting the self-polarization rotation in a SOA structure, using the same setup configuration. The implementation of these all-optical signal processing functions is based on experimental investigation in free space space in order to allow optimum control and preserve the polarization state of the injected and collected signals. Switching between these functions is done by only adjusting the input optical

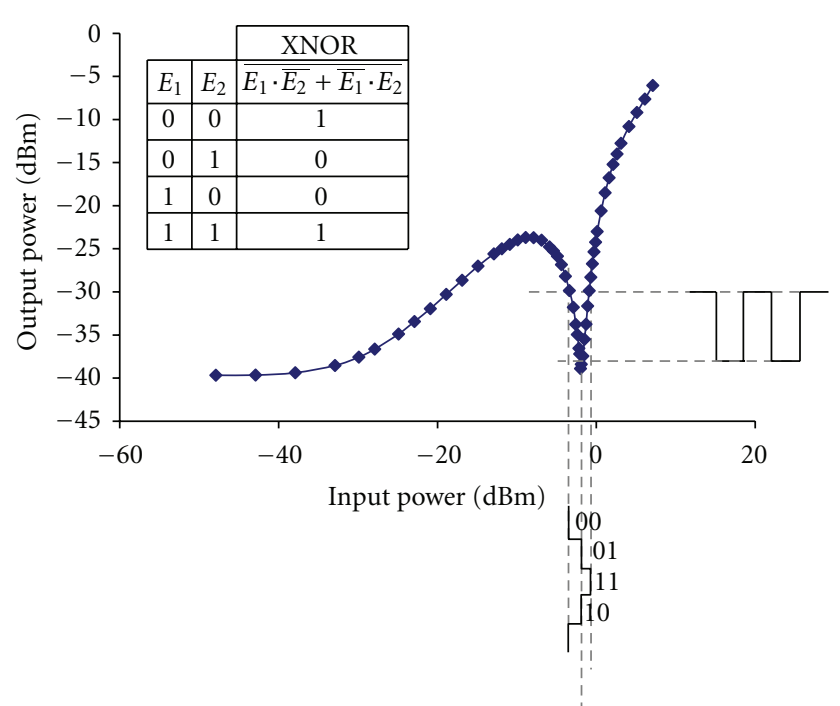

Figure 8: Measured static transfer function and principle of operation for XNOR function at a blocking power equal to $-2 \mathrm{dBm}$ and wavelength equal to $1550 \mathrm{~nm}$.

power level. Since each one of the proposed functions can be applied to various networking applications, they will play an important role in future high-capacity optical communication networks. This study can be extended to exploit the obtained static response of the SOA using the SPR and demonstrate its capabilities in the dynamic regime.

\section{Acknowledgments}

The authors would like to thank the team of RESO lab, EA 3380, ENIB, Brest, France: A. Sharaiha, M. Guégan, P. Morel, and F. Bentivegna for their interest, support, fruitful discussions, and cooperation and M. Tariaki for his help to complete the experiments.

\section{References}

[1] J. Leuthold, B. Mikkelsen, G. Raybon et al., "All-optical wavelength conversion between 10 and $100 \mathrm{~Gb} / \mathrm{s}$ with SOA delayedinterference configuration," Optical and Quantum Electronics, vol. 33, no. 7-10, pp. 939-952, 2001.

[2] Y. Said and H. Rezig, "Semiconductor optical amplifier nonlinearities and their applications for next generation of optical networks," in Advances in Optical Amplifiers, P. Urquhart, Ed., chapter 2, pp. 27-52, 2011, http//www.intechopen.com/articles/show/title/semiconductor-optical-amplifier-nonlinearities-and their-applications-for-next-generationof-optical.

[3] R. Randhawa, S. Singh, J. S. Sohal, and R. S. Kaler, "Wavelength converter using semiconductor optical amplifier MachZehnder interferometer based on XPM at $40 \mathrm{~Gb} / \mathrm{s}$ for future transport networks," Fiber and Integrated Optics, vol. 28, no. 2, pp. 154-169, 2009.

[4] M. Spyropoulou, N. Pleros, K. Vyrsokinos et al., "40 Gb/s NRZ wavelength conversion using a differentially-biased SOA-MZI: theory and experiment," Journal of Lightwave Technology, vol. 29, no. 10, pp. 1489-1499, 2011. 
[5] L. Bramerie, M. Gay, J. C. Simon et al., "A versatile optical spot-size converter desig," in Proceedings of the European Conference on Optical Communications (ECOC '04), vol. We2.5.2, pp. 468-469, Stockholm, Sweden, 2004.

[6] Y. Dong, Z. Li, C. Lu, Y. Wang, and T. H. Cheng, "3R all-optical regeneration and wavelength conversion based on cross polarization modulation effect from a single semiconductor optical amplifier," in Proceedings of the 16th IEEE LEOS Annual Meeting Conference Proceedings, pp. 403-404, October 2003.

[7] S. Philippe, A. L. Bradley, B. Kennedy, F. Surre, and P. Landais, "Experimental investigation of polarization effects in semiconductor optical amplifiers and implications for alloptical switching," Journal of Lightwave Technology, vol. 26, no. 16, pp. 2977-2985, 2008.

[8] J. Dong, X. Zhang, J. Xu, and D. Huang, " $40 \mathrm{~Gb} / \mathrm{s}$ alloptical logic NOR and OR gates using a semiconductor optical amplifier: experimental demonstration and theoretical analysis," Optics Communications, vol. 281, no. 6, pp. 1710$1715,2008$.

[9] J. Y. Kim, J. M. Kang, T. Y. Kim, and S. K. Han, "Alloptical multiple logic gates with XOR, NOR, OR, and NAND functions using parallel SOA-MZI structures: theory and experiment," Journal of Lightwave Technology, vol. 24, no. 9, pp. 3392-3399, 2006.

[10] J. Zhang, J. Wu, C. Feng, K. Xu, and J. Lin, "All-optical logic or gate exploiting nonlinear polarization rotation in an SOA and red-shifted sideband filtering," IEEE Photonics Technology Letters, vol. 19, no. 1, pp. 33-35, 2007.

[11] T. Durhuus, B. Mikkelsen, C. Joergensen, S. L. Danielsen, and K. E. Stubkjaer, "All-optical wavelength conversion by semiconductor optical amplifiers," Journal of Lightwave Technology, vol. 14, no. 6, pp. 942-954, 1996.

[12] S. Fu, J. Dong, P. Shum, L. Zhang, X. Zhang, and D. Huang, "Experimental demonstration of both inverted and noninverted wavelength conversion based on transient cross phase modulation of SOA," Optics Express, vol. 14, no. 17, pp. 75877593, 2006.

[13] A. Matsumoto, K. Nishimura, K. Utaka, and M. Usami, "Operational design on high-speed semiconductor optical amplifier with assist light for application to wavelength converters using cross-phase modulation," IEEE Journal of Quantum Electronics, vol. 42, no. 3, Article ID 01597418, pp. 313-323, 2006.

[14] C. Politi, D. Klonidis, and M. J. O’Mahony, "Dynamic behavior of wavelength converters based on FWM in SOAs," IEEE Journal of Quantum Electronics, vol. 42, no. 2, pp. 108125, 2006.

[15] S. Jansen, G. Khoe, H. de Waardt et al., "Optimizing the wavelength configuration for FWM-based demultiplexing in a SOA," in Proceedings of the Optical Fiber Communication Conference (OFC'03), pp. 539-541, 2003.

[16] J. P. Turkiewiez, J. J. V. Olmos, A. M. J. Koonen, G. D. Khoe, and H. de Waardt, "All-optical 1310-to-1550 nm wavelength conversion including transmission over two fibre links," in Proceedings of the IEEE/LEOS 10th Annual Symposium Benelux Chapter, pp. 189-192, Mons, Belgium, December 2005.

[17] M. Tariaki, A. Sharaiha, M. Guégan, F. F. L. Bentivegna, and M. Amaya, "All-optical inverted and non-inverted wavelength conversion based on cross polarization modulation in a semiconductor optical amplifier," in Proceedings of the $3 \mathrm{rd}$ International Conference on Information and Communication
Technologies: From Theory to Applications (IEEE-ICTTA '08), pp. 1-6, Damascus, Syria, April 2008.

[18] A. Sharaiha, J. Topomondzo, and P. Morel, "All-optical logic AND-NOR gate with three inputs based on cross-gain modulation in a semiconductor optical amplifier," Optics Communications, vol. 265, no. 1, pp. 322-325, 2006.

[19] A. Hamie, A. Sharaiha, and M. Guegan, "Demonstration of an all-optical logic OR gate using gain saturation in an SOA," Microwave and Optical Technology Letters, vol. 39, no. 1, pp. 39-42, 2003.

[20] G. Berrettini, A. Simi, A. Malacarne, A. Bogoni, and L. Poti, "Ultrafast integrable and reconfigurable XNOR, AND, NOR, and NOT photonic logic gate," IEEE Photonics Technology Letters, vol. 18, no. 8, pp. 917-919, 2006.

[21] Z. Li and G. Li, "Ultrahigh-speed reconfigurable logic gates based on four-wave mixing in a semiconductor optical amplifier," IEEE Photonics Technology Letters, vol. 18, no. 12, pp. 1341-1343, 2006.

[22] L. Han, X. Teng, L. Hu, N. Hua, and H. Zhang, "All optical NOR and or gates using cross polarization modulation in a single SOA," in Proceedings of the 18th Annual Meeting of the IEEE Lasers and Electro-Optics Society (LEOS '05), pp. 438439, October 2005.

[23] J. Zhang, J. Wu, C. Feng, K. Xu, and J. Lin, "40 Gbit/s alloptical logic NOR gate based on nonlinear polarisation rotation in SOA and blue-shifted sideband filtering," Electronics Letters, vol. 42, no. 21, pp. 1243-1244, 2006.

[24] Y. Liu, M. T. Hill, E. Tangdiongga et al., "Wavelength conversion using nonlinear polarization rotation in a single semiconductor optical amplifier," IEEE Photonics Technology Letters, vol. 15, no. 1, pp. 90-92, 2003.

[25] B. F. Kennedy, S. Philippe, F. Surre, A. L. Bradley, and P. Landais, "Investigation of optimum wavelength converter based on nonlinear polarisation rotation in a bulk SOA," IET Optoelectronics, vol. 1, no. 2, pp. 55-60, 2007. 

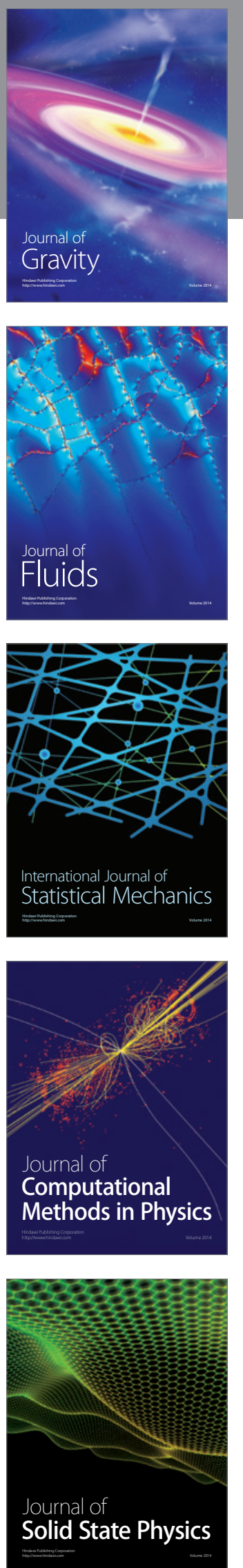

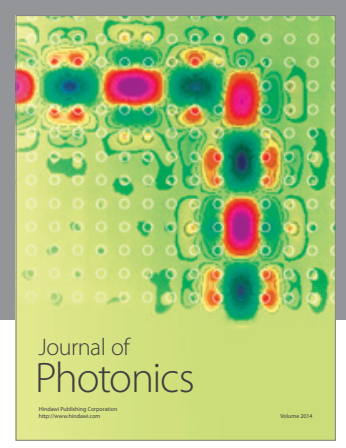

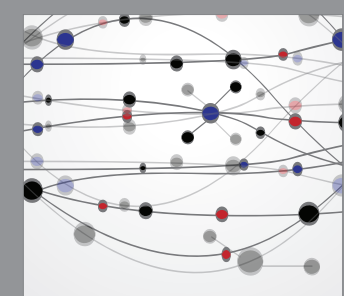

The Scientific World Journal
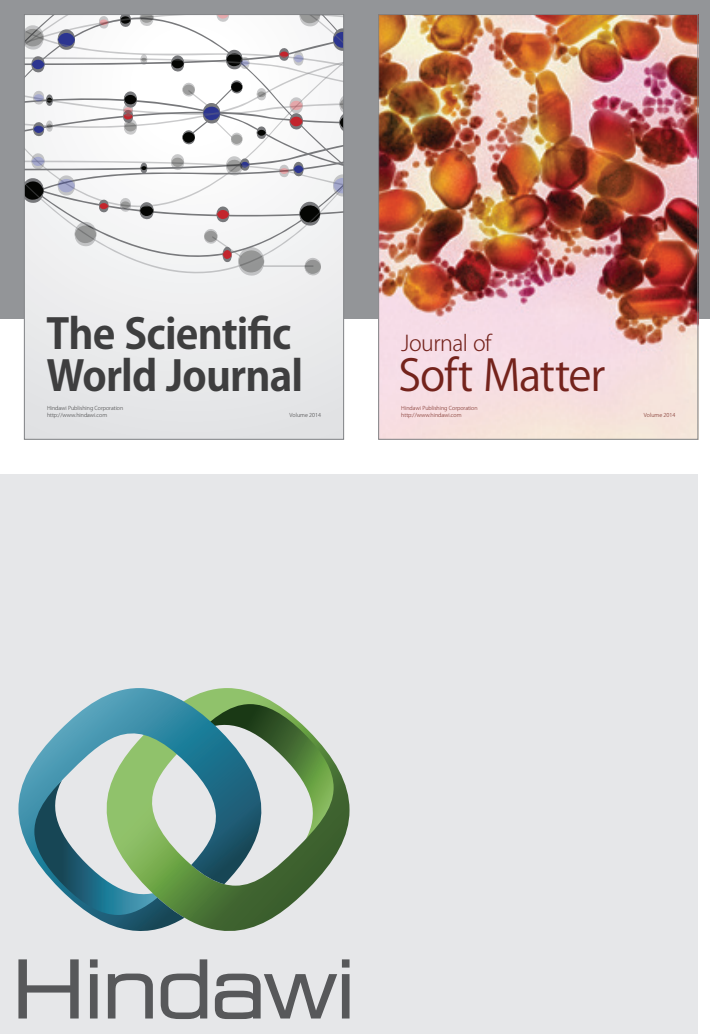

Submit your manuscripts at

http://www.hindawi.com
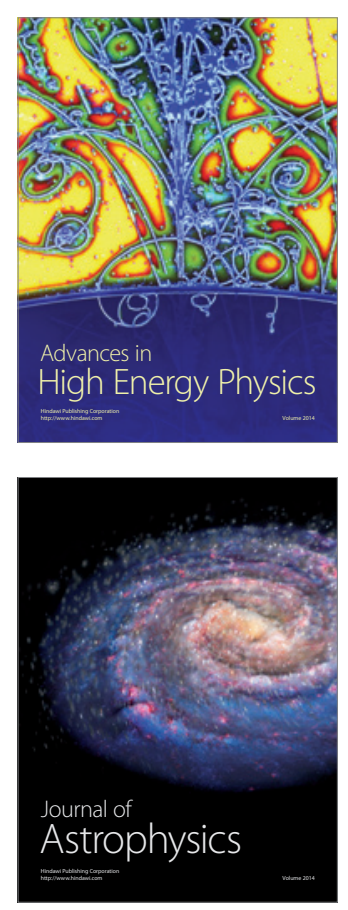
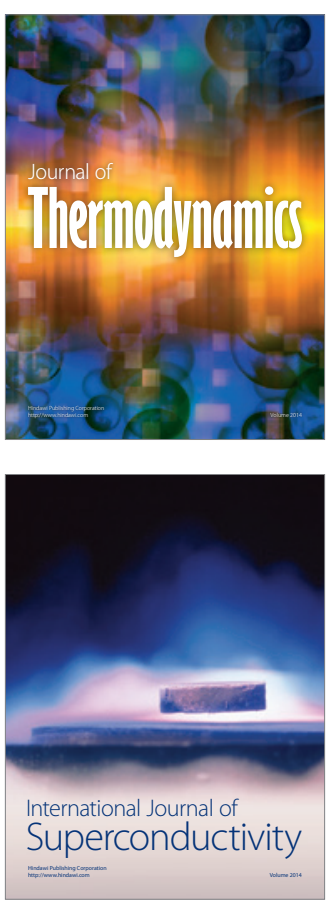
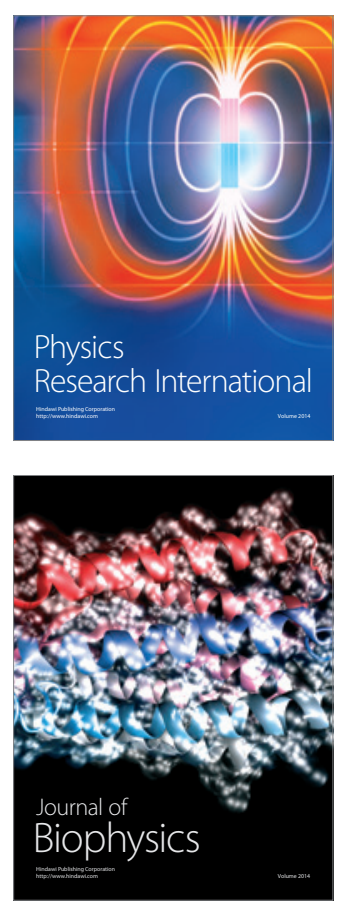
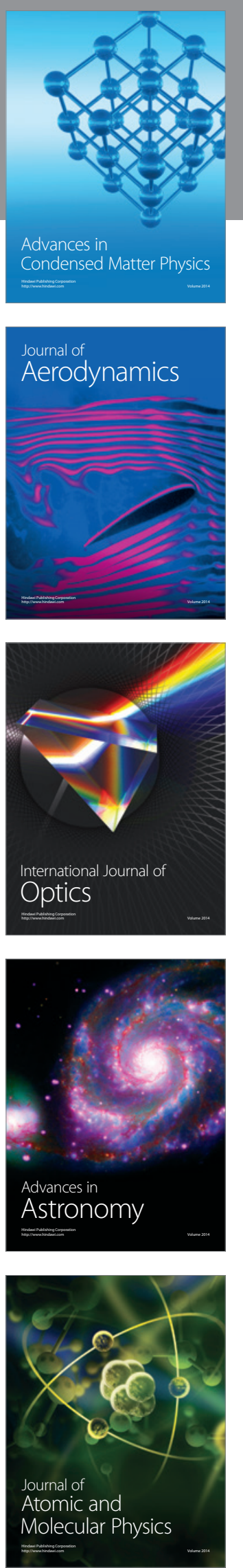